\title{
The evaluation of energy saving performance for the modular design centrifugal chiller
}

\author{
YoonJei Hwang ${ }^{1, *}$, HanYoung Park ${ }^{1}$, SaiKee $\mathrm{Oh}^{1}$, HoRim Lee ${ }^{2}$, YoungSu Park $^{2}$, and KamGyu Lee ${ }^{2}$ \\ ${ }^{1}$ Air Solution Laboratory, LG Electronics Inc., 150-802 Seoul, Korea \\ ${ }^{2}$ Air Solution Business Unit, LG Electronics Inc., 642-713 Changwon City, Korea
}

\begin{abstract}
The modular concept design and operation of a centrifugal chiller offers the potential of increased cycle efficiency both at full load and off load conditions compared to the single compressor chiller. Modular chiller design is that a smaller, individual chiller can be connected with another, to make a larger capacity system. In the case of a tandem chiller, there would be faced to the higher initial cost or bigger foot print than a single compressor chiller at the same capacity because manufacturing cost, weight and size will be decreased according to increase of the refrigeration capacity. Therefore, it is obvious that a modular chiller have to be improved its efficiency first by both the operation algorithm and major cycle components. Eventually, the efficiency of the modular chiller achieves up to $6 \%$ at the full load condition after added series counter flow effect as much as $3 \%$ and more $3 \%$ added by improved aerodynamic design for impeller.Moreover, maximize off design potential through the capacity combination algorithm as the way of uneven loading makes $24 \%$ improved based on AHRI 550/590.
\end{abstract}

\section{Introduction}

The modular concept centrifugal chiller offers easy to capacity expansion, better efficiency and less occufy by the help of technogies, where from the strategy for capacity combination, a smarter operation algorithm and implemented a couple of high efficient components. Modular chiller design is that a smaller, individual chiller can be connected with another, to make a larger capacity system by the ways of parallel or serial combination. In the case, two basic modules, which are 300 and 500RT, bring a good expandability from 300RT to $2,400 \mathrm{RT}$. Each basic module has the same mechanical dimensions except impellers, 300RT module can be expanded up to 250 350RT and 400 600RT by 500RT module. Eventually, there have six capacity modules. Customers are available 2,400RT as a maximum capacity if it be connected with four 600RT modules. Figure 1 shows that the picture of 1,000RT by combination of two 500RT module. It is obviously that combine two chillers to make as a one body increases installation area, which could be more than double due to

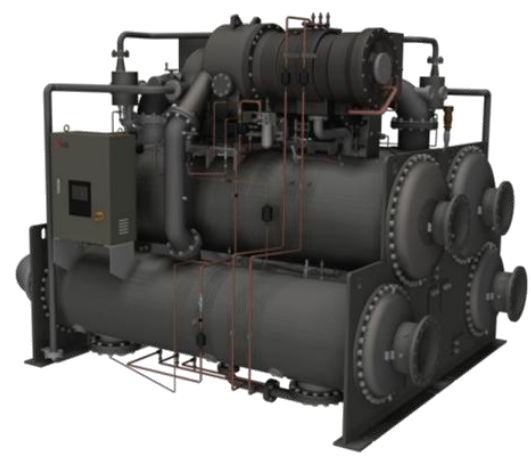

Fig. 1. Modular concept centrifugal chiller. water piping arrangement as a worst case. The modular design takes an advanced geometric shape by stack them until it maintained stable structure. By the help of hexagonal arrangement, modular design saves 30\% installation area if it compared to two chillers installed at same capacity. Most of service parts are arranged at the same side of modular chillers for easy to service. There are two practical configurations for multiple chiller application, which can be a serial and parallel way through the same basic module. The serial connection is a basic even it can be installed as a parallel way. Basic modules with different capacity can be combined to take better part load performance or get better load matching with customer building. Figure 2 is an example for the case of 800RT combination.
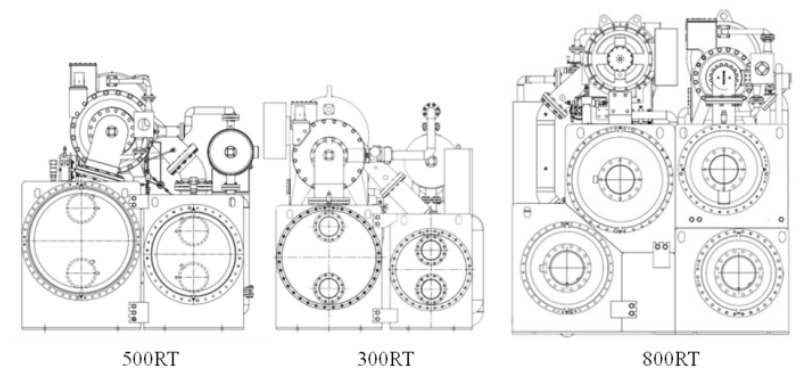

Fig. 2. The comparison of installation between the two single compressor chillers and the module chiller.

Figure 3 shows that the concept of modular chiller. A series counter flow cycle brings the efficiency benefits, there can be found more 3\% higher efficiency at Korea 


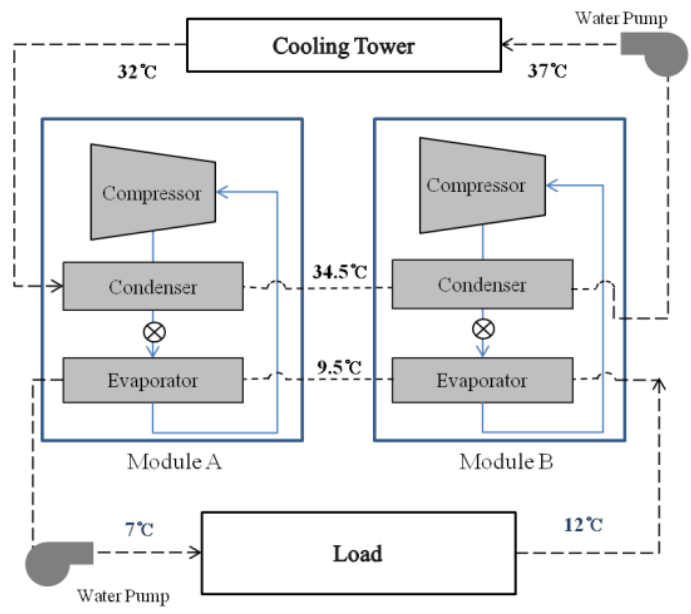

Fig. 3. The schematics of modular concept chiller system.

standard condition due to 2.5 Celsius degree reduction of compressor duty than any stand alone cycle. However, it would be not easy to spread out the benefit for more than four modules because manufacturing cost and foot print becomes worse than a single compressor system. So, the modular chiller has to be designed by smart ways, where the most of the components are designed efficiently, easy to access to all service components for servicibility by located it at the same side and get less foot print by staggered arrangement to save lands for commercial purposes.

\section{Technologies for the modular concept design}

The initial cost of a single compressor centrifugal chiller would be a little bit cheaper than a tandem compressor chiller at the same capacity base. There should be not much attractive to the customers who want to buy modular chiller while there have no cost benefits. Cost effective design has been done to get cost competitiveness, where from updated impeller design, minimize pressure drop of flow paths by optimize refrigerant flow at the connecting tubes and take out duplicated components thanks by the module combination. It was 3\% improvement of efficiency for impeller where from newly designed shape of blades together with enhanced seal [1]. Figure 4 shows the effect of Mach number improvement.
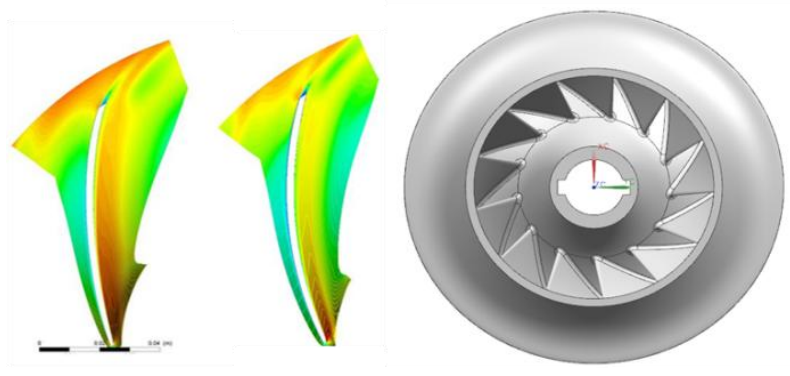

Fig. 4. Mach number contour for the updated impeller.
For heat exchangers, eliminate sub cooler at the bottom of condenser because double stage cycle has economizer, there not much need to make degree of sub cooling at the inlet of expansion device. Moreover, economizer be moved from the outside of condenser to the room, where coming from elimination of sub cooler at the inside of condenser as be showed at figure 5 .

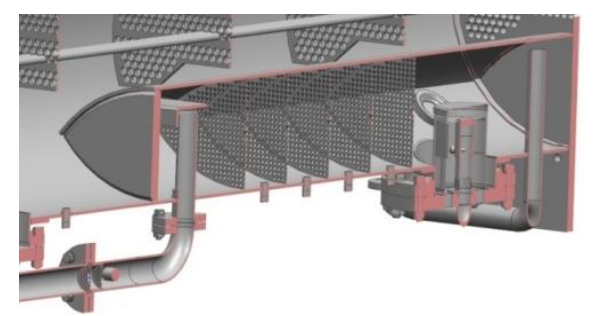

Fig. 5. Economizer located at the bottom of condenser shell.

A couple of connection tubes be eliminated, it brings improved system efficiency by decreased pressure drops between connection tubes, the cost of manufacturing also becomes cheaper than previous design. The dimensions of all base modules, which are the shell diameter and length, are all the same for simplification and standardization while tube arrays and the number of tubes could be changed depend on design capacities. One of key technology for modular chiller is that the operation strategies, especially for how to manage compressors to the way of getting higher part load efficiency and less electrical installation. There should be take benefits for energy efficiency if a chiller plant be managed as running minimum compressors to get the same cooling capacity. The modular chiller offers a single water flow pass to avoid fell into unstable cycle at the condition of a different capacity compressors, which could be shown different LTD (least temperature difference) between two different cycles. The multiple compressor running with different capacities brings positive results as much as $24 \%$ better than to a multiple compressor with the same capacity, as shown in table 1 .

Table 1. The full and part load efficiencies(COP) according to the installation type.

\begin{tabular}{|c|c|c|c|c|}
\hline \multicolumn{2}{|c|}{$\begin{array}{c}\text { Installation } \\
\text { type of chiller }\end{array}$} & $\begin{array}{c}\text { Chiller with a } \\
\text { single } \\
\text { compressor }\end{array}$ & $\begin{array}{c}\text { Chiller with a } \\
\text { dual } \\
\text { compressor }\end{array}$ & Modular \\
\hline Load(\%) & $\begin{array}{c}\text { Capacity } \\
\text { (RT) }\end{array}$ & $800 \mathrm{RT}$ & $\begin{array}{c}\text { A 400RT/ } \\
\text { B 400RT }\end{array}$ & $\begin{array}{c}\text { A 500RT/ } \\
\text { B 300RT }\end{array}$ \\
\hline $\mathbf{1 0 0 \%}$ & $\mathbf{8 0 0}$ & 6.31 & 6.47 & 6.67 \\
\hline $75 \%$ & 600 & 7.20 & 7.38 & 7.87 \\
\hline $50 \%$ & 400 & 7.58 & 9.41 & 9.96 \\
\hline $25 \%$ & 200 & 6.17 & 6.96 & 9.11 \\
\hline \multicolumn{6}{|c|}{ IPLV } & 7.23 & 8.23 & 8.95 \\
\hline
\end{tabular}

In modular chillers, a master module keeps on monitoring and gathering data from each chiller module through interworking communication network as to acting as one system. Target operation point be found through the target loads and the target compression ratio, 
which be calculated based on the target chilled water outlet temperature and the current inlet temperature of water together. Once be found the target point, algorithm for the compressor sequence guides that how many compressors to be run to minimize energy consumption. The control logic becomes important to multiple compressor system because there could be seen different results depends on how many compressors have run even at the same operation condition. Especially for part load condition less than $50 \%$, energy saving could be better if less compressors are running as much as system can handle. The conventional algorithm, which is consider for target setting by only the outlet chilled water temperature without considering compressor sequence, IGVs will be closed to find the target chilled water temperature by the same degree of opening to all compressors when building load is getting decreased. The results should be better when the system uses the sequence algorithm for load matching than doing it without the sequence algorithm.

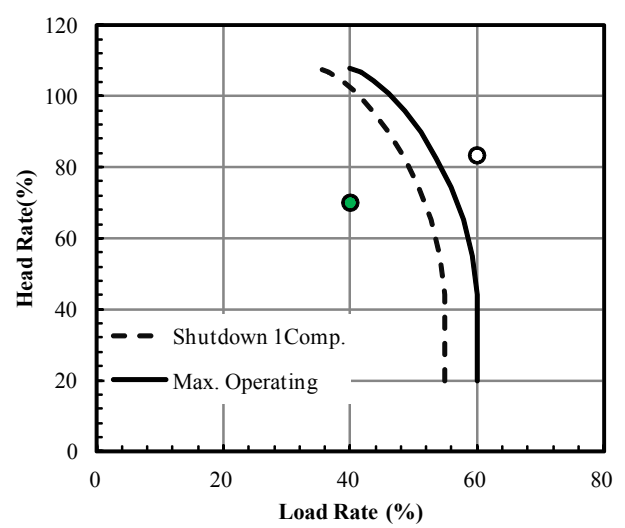

Fig. 6. The sequence algorithm to make decide the number of running compressors.

Watching together with the head rate and load rate to find a current position and the target position at the same time, then make decide how many compressors should be running by the lines at shown in figure 6 . The algorithm begins to calculate the number of compressors when the operation point are approach to the broken line. Once current condition be satisfied multiple compressor running condition, the algorithm begins to tracking the electric current toward all running compressors to share the same loads through keeps it the same electric current. Moreover, it is available to alternating operation for compressors to the purpose of managing uniform operation time. Table 2 shows how works compressor on/off algorithm depend on loads.

Table 2. The load matching strategy according to algorithm

\begin{tabular}{|c|c|c|c|c|}
\hline \multirow{2}{*}{$\begin{array}{c}\text { Load } \\
(\%)\end{array}$} & \multicolumn{2}{|c|}{ Conventional } & \multicolumn{2}{c|}{$\begin{array}{c}\text { Compressor sequence } \\
\text { algorithm }\end{array}$} \\
\cline { 2 - 5 } & Lead & Lag & Lead & Lag \\
\hline 100 & 100 & 100 & 100 & 100 \\
\hline 50 & 50 & 50 & 50 & 50 \\
\hline 25 & 25 & 25 & 50 & 0 \\
\hline
\end{tabular}

\section{Benefits of modular concept design}

Modular chiller provides higher limitation to the electric currents by through serial time lag start up. There's no need to start it up at one time until to the nominal electric capacity if the time lag between motors can be set as not too long because the serial start up can save an electric power to the system loads. Figure 7 shows that the serial start up of the system through a direct current took a lower current limitation level than one time start up by a reactor system at 6,600 volt. The benefits will be increased according to more number of motors. In case of 2,000RT, there happened a big difference than $1,000 \mathrm{RT}$ because saving effects can be taken more according to increase of the number of compressor motors. It definitely let both the electric power and control management system saves investment as much as number of modules.

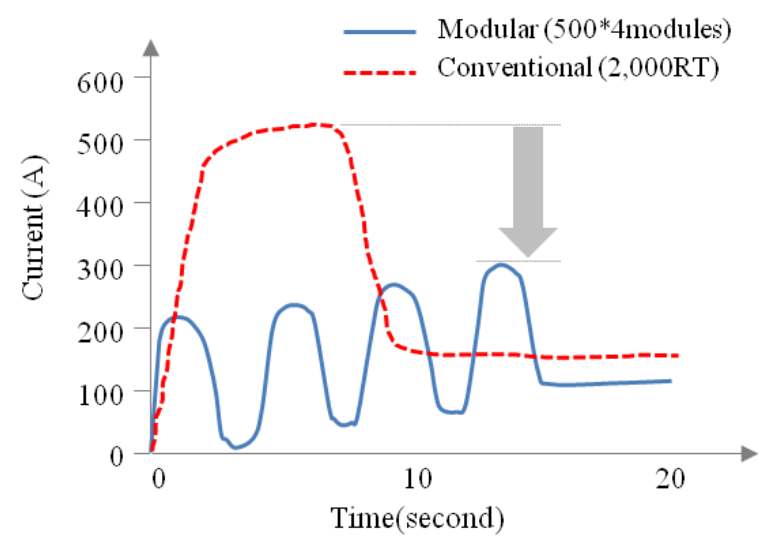

Fig. 7. The comparison of the limitation of an electric current according to the ways of start up.

Table 3 says how much money can saved in terms of an electrical investment if customer takes the module chiller system. Comparisons has been done at the based on reactor type as a conventional besides at the based on direct type to modular chiller. Installation cost for the electricity was cheaper to the conventional, but total cost for electricity is better to the modular chiller because the transformer capacity becomes smaller, which is cheaper, than that of the conventional chiller.

Table 3. The examples of an investment to the electricity

\begin{tabular}{|c|c|c|}
\hline \multirow{2}{*}{} & \multicolumn{2}{|c|}{ Types of chiller } \\
\cline { 2 - 3 } & Conventional & Modular \\
\hline Start up type & reactor & direct \\
\hline $\begin{array}{c}\text { Start up Panel } \\
\text { Investment }\end{array}$ & 14,000 & 12,700 \\
\hline Cost of Electricity & 3,000 & 9,100 \\
\hline $\begin{array}{c}\text { Transformer } \\
\text { Investment }\end{array}$ & 73,200 & 45,700 \\
\cline { 2 - 3 } & $(3,000 \mathrm{KVA})$ & $(1,750 \mathrm{KVA})$ \\
\hline Total(USD) & 90,200 & 67,500 \\
\hline
\end{tabular}

The installation flexibility be added as another benefit from module chiller. There have a couple of installation ways depends on the space availability of a building, 
which are parallel and serial combination as shown at the figure 8 .

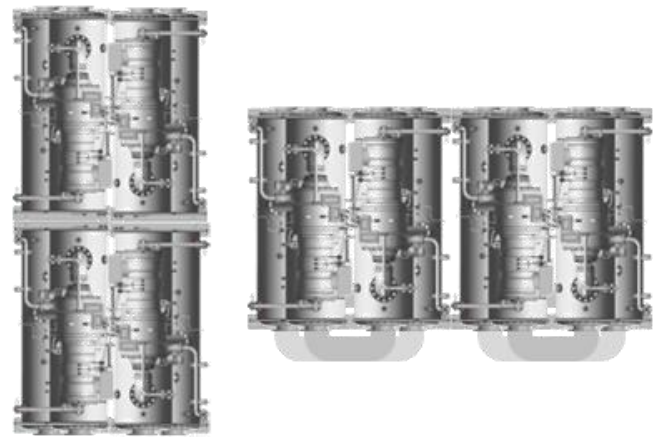

Fig. 8. The installation flexibility of the modular chiller (four modules make the rating capacity).

The modular chiller brings 3\% higher COP at full load condition than any conventional stand alone chiller due to the cycle effect by the series counter flow at AHRI condition. To maximize energy performance, combine modules with a different capacity, which could be a $300 \mathrm{RT}$ and a 500RT to make $800 \mathrm{RT}$, it also brings $24 \%$ improvement on IPLV as shown in table2. However, uneven module matching could be shown cycle unstable if not uses load matching sequence algorithm together. All of performance improvements can be converted to USD by calculation. Table 4 shows how much money can be saved by 800RT module with different capacities versus conventional chiller. It based on the typical water temperature conditions, which were $12^{\circ} \mathrm{C}$ to $7^{\circ} \mathrm{C}$ at chilled water inlet/outlet and $32^{\circ} \mathrm{C}$ to $27^{\circ} \mathrm{C}$ at cooling water inlet/outlet. There are many kinds of patterns to operate chillers according to the demands from sites, the module chiller, which be implemented compressor sequence algorithm, provides a good energy performance for the sites, especially where be required the higher performance to part loads during operation mainly. Inverter driver would be one of good solution to the case, however, it becomes not a good solution to where the site only has a high voltage current because the site has to be converted their high voltage to the low voltage through transformer, which is very expensive.
Moreover, the cost of electricity should to be added. Another case would be a customer who willing to buy a centrifugal chiller which has an induction motor with inverter as starter. There can be seen many of inverter centrifugal chillers with induction motor in the industry even the centrifugal compressor has a lot of huddles to use inverter benefits. It has been introduced VRF industry first, user experience says it definitely brings energy saving. However, a centrifugal compressor is not a volumetric compression type besides VRF did. A centrifugal compressor is a type of dynamic compressor with a radial design. Unlike volumetric compressors that work at a constant flow, dynamic compressors work at a constant pressure and the performance is affected by external conditions such as changes operation heads. The rpm changes by inverter to the centrifugal compressor works on both the mass flow rate of refrigerant and the compression ratio besides only it changes mass flow rate without compression ratio to the volumetric compression. The benefits by inverter should be different from the condition of operation heads at the site. It maximized at the AHRI condition by AHRI 550/590, which be changed outdoor temperatures a lot according to the changes of the building loads. The CCET (Constant Cooling Entering Temperature) condition, which not changed outdoor temperatures at all, shows a minimum impact on IPLV by inverter because operation points at

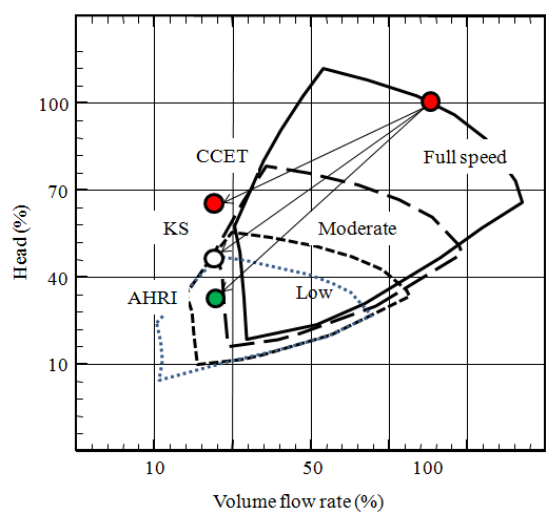

Fig. 9. The relationship between AHRI and other load points and compressor envelopes moving according to rpm.

Table 4. The analysis for the electric charges according to the type of chiller at 800RT by kepco.co.kr [2]

\begin{tabular}{|c|c|c|c|c|c|c|c|c|c|c|c|c|c|}
\hline \multicolumn{2}{|c|}{$800 \mathrm{RT}$} & \multicolumn{6}{|c|}{ Stand alone single compressor with Inverter drive } & \multicolumn{6}{|c|}{ Modular Concept } \\
\hline & & \begin{tabular}{|c|} 
Power \\
Consumption \\
$(\mathrm{kW})$
\end{tabular} & Months & Hours & $\begin{array}{c}\text { Base } \\
\text { Charge } \\
(\$ / \mathrm{kW})\end{array}$ & \begin{tabular}{|l|} 
Electric \\
Charge \\
$(\$ / \mathrm{kWh})$
\end{tabular} & $\begin{array}{c}\text { Total } \\
\text { Electric } \\
\text { Charge (USD) }\end{array}$ & $\begin{array}{c}\text { Power } \\
\text { Consumption } \\
(\mathrm{kW})\end{array}$ & Months & s Hours & \begin{tabular}{|c} 
Base \\
Charge \\
$(\$ / k W)$
\end{tabular} & $\begin{array}{l}\text { Electric } \\
\text { Charge } \\
(\$ / k W h)\end{array}$ & $\begin{array}{c}\text { Total } \\
\text { Electric } \\
\text { Charge (USD) }\end{array}$ \\
\hline $\begin{array}{c}\text { Base charge } \\
\text { for chiller }\end{array}$ & $\begin{array}{c}\text { Percent of } \\
\text { load(\%) }\end{array}$ & 481.6 & 12 & & 8.92 & & 51,540 & 455.2 & 12 & & 8.92 & & 48,715 \\
\hline \multicolumn{2}{|c|}{ Summer } & & & & & & 100,352 & & & & & & 94,851 \\
\hline Low Load & 100 & 481.6 & & 920 & & 0.050 & 22,234 & 455.2 & & 920 & & 0.050 & 21,015 \\
\hline Middle Load & 100 & 481.6 & & 736 & & 0.099 & 34,930 & 455.2 & & 736 & & 0.099 & 33,015 \\
\hline Full Load & 100 & 481.6 & & 552 & & 0.162 & 43,187 & 455.2 & & 552 & & 0.162 & 40,820 \\
\hline \multicolumn{2}{|c|}{ Spring/Autumn } & & & & & & 52,816 & & & & & & 43,420 \\
\hline Low Load & 25 & 121.8 & & 1530 & & 0.050 & 9,352 & 82.4 & & 1530 & & 0.050 & 6,330 \\
\hline Middle Load & 50 & 198.4 & & 1224 & & 0.070 & 17,065 & 150.8 & & 1224 & & 0.070 & 12,967 \\
\hline Full Load & 75 & 313.2 & & 918 & & 0.092 & 26,399 & 286.2 & & 918 & & 0.092 & 24,124 \\
\hline \multicolumn{2}{|c|}{ Winter } & & & & & & 40,042 & & & & & & 28,779 \\
\hline Low Load & 25 & 121.8 & & 1200 & & 0.057 & 8,305 & 82.4 & & 1200 & & 0.057 & 5,621 \\
\hline Middle Load & 25 & 121.8 & & 960 & & 0.099 & 11,544 & 82.4 & & 960 & & 0.099 & 7,814 \\
\hline Full Load & 50 & 198.4 & & 720 & & 0.141 & 20,194 & 374.3 & & 720 & & 0.141 & 15,344 \\
\hline \multicolumn{2}{|c|}{ Total } & & & 8760 & & & 244,750 & & & 8760 & & & 215,765 \\
\hline
\end{tabular}


a part load can be departs from the envelope of compressor easily. The benefit at the condition of KS (Korea Standard) shrank up even there are not much gaps of outdoor temperatures according to the part load, which is $32^{\circ} \mathrm{C}$ at the full load and $27^{\circ} \mathrm{C}$ at $0 \%$ load. The figure 9 shows the relationship between the operation points at each load and the compressor envelopes by the changes of motor rpms. In case of a stand-alone chiller with inverter, the system could not meet a required operation point when the required load becomes too low even the system tried to match the point by reduce rpm. However, the modular chiller has relatively enough margin to low loads. As you can see figure 10, each module is running as a full capacity together to meet the required load, which becomes $100 \%$ load, with maintaining required head. Modular chiller can meets the $25 \%$ load by use two compressor operation, which becomes $50 \%$ operation at one module, besides a standalone one compressor chiller with inverter try to meet the same point by reducing $\mathrm{rpm}$ and closing IGV together as much as it can do until surge, but finally failed [3].

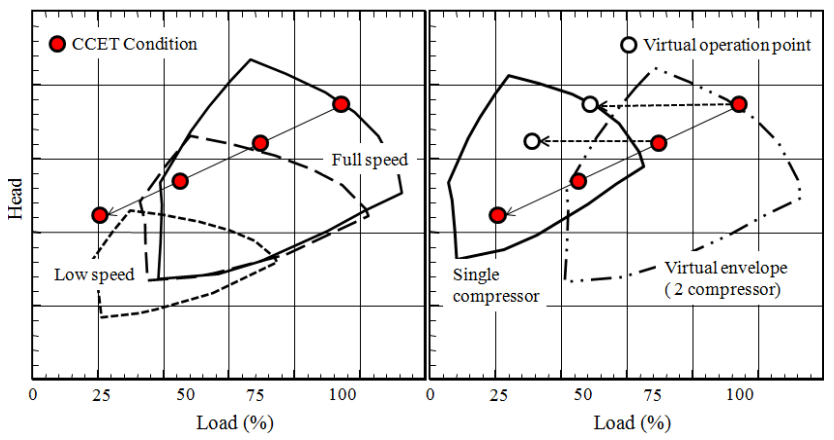

Fig. 10. The load matching strategy for a modular chiller with two compressors.

Modular chiller has more options to create an opportunities to operate chiller in a different, but efficient manner at all time than mostly stand alone one compressor chiller with inverter. The analysis says that there have lots of results depends on what's the operation condition at sites. From the cycle point of view, the modular chiller is most efficient at $50 \%$ load, where only one module is running because heat exchangers becomes twice the size than that of normally used for the compressor size. Moreover, running by one module leads that the operation point stays very near to the best aerodynamic efficient point of impeller. Assuming the site have seasonal load, where it mixed with three kinds of representative load like AHRI, KS and CCET. The efficiencies of the three types of system be measured at the representative load conditions at the calorimeter as shown at the table 5. The calculation results are shown at the table 6. Modular concept and a stand-alone chiller with inverter systems are always better efficiency than a constant speed one compressor chiller system as much as $10.7 \%$ as a maximum. The two types of chiller, which are modular concept and a stand-alone chiller with inverter, looks not much different in terms of efficiency, but the modular concept chiller should be the better option for the sites, where has higher head with normally operated at the low load conditions.

Table 5. The analysis for the electric charges according to the type of chiller at 1,000RT by kepco.co.kr

\begin{tabular}{|c|c|c|c|c|}
\hline \multirow{2}{*}{ Seasonal Band } & \multirow{2}{*}{ Operation Condition } & \multicolumn{3}{|c|}{ Hour Band } \\
\cline { 3 - 5 } & & Full & Middle & Low \\
\hline Summer (June $\sim$ Aug.) & CCET & $100 \%$ & $75 \%$ & $50 \%$ \\
\hline Spring/Autumn & KS & $75 \%$ & $50 \%$ & $25 \%$ \\
\hline Winter (Nov. $\sim$ Feb.) & AHRI & $75 \%$ & $50 \%$ & $25 \%$ \\
\hline
\end{tabular}

\begin{tabular}{|c|c|c|c|c|c|c|c|c|c|}
\hline \multirow[t]{2}{*}{ Load } & \multicolumn{3}{|c|}{$\begin{array}{l}\text { Stand alone single } \\
\text { compressor with } \\
\text { Inverter }(\mathrm{kW} / \mathrm{RT})\end{array}$} & \multicolumn{3}{|c|}{$\begin{array}{l}\text { Modular Concept } \\
\quad(\mathrm{kW} / \mathrm{RT})\end{array}$} & \multicolumn{3}{|c|}{$\begin{array}{l}\text { Stand Alone single } \\
\text { compressor W/O } \\
\text { Inverter }(\mathrm{kW} / \mathrm{RT})\end{array}$} \\
\hline & AHRI & KS & CCET & AHRI & $\mathrm{KS}$ & 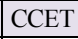 & AHRI & KS & CCET \\
\hline $00 \%$ & 0.605 & 0.605 & 060 & & & & & \begin{tabular}{|l|l|}
0.587 \\
\end{tabular} & \\
\hline $75 \%$ & 0.461 & & & & & & & & \\
\hline $50 \%$ & & & & & & & & & \\
\hline $25 \%$ & 366 & 1.076 & 1.135 & 0.520 & 0.635 & 0.670 & \begin{tabular}{|l|}
0.687 \\
\end{tabular} & 1.029 & 1.084 \\
\hline
\end{tabular}

Table 6. The efficiencies of the three types of system at various load condition

\begin{tabular}{|c|c|c|c|c|c|c|c|c|c|c|c|}
\hline \multicolumn{2}{|c|}{$1000 \mathrm{RT}$} & \multicolumn{6}{|c|}{ Stand alone single compressor with Inverter drive } & \multicolumn{2}{|c|}{ Modular Concept } & \multicolumn{2}{|c|}{$\begin{array}{l}\text { Stand Alone single } \\
\text { compressor W/O inverter }\end{array}$} \\
\hline & & \begin{tabular}{|c|} 
Power \\
Consumption \\
$(\mathrm{kW})$ \\
\end{tabular} & Months & Hours & $\begin{array}{c}\text { Base } \\
\text { Charge } \\
(\$ / \mathrm{kW}) \\
\end{array}$ & \begin{tabular}{|l} 
Electric \\
Charge \\
$(\$ / \mathrm{kWh})$ \\
\end{tabular} & \begin{tabular}{|c||} 
Total \\
Electric \\
Charge (USD) \\
\end{tabular} & $\begin{array}{c}\text { Power } \\
\text { Consumption } \\
(\mathrm{kW}) \\
\end{array}$ & \begin{tabular}{|c|} 
Total \\
Electric \\
Charge (USD) \\
\end{tabular} & \begin{tabular}{|c|} 
Power \\
Consumption \\
$(\mathrm{kW})$ \\
\end{tabular} & $\begin{array}{c}\text { Total } \\
\text { Electric } \\
\text { Charge (USD) } \\
\end{array}$ \\
\hline $\begin{array}{l}\text { Base charge } \\
\text { for chiller }\end{array}$ & $\begin{array}{c}\text { Percent of } \\
\text { load(\%) }\end{array}$ & 604.7 & 12 & & 8.92 & & 64,715 & 558.0 & 59,716 & 587.1 & 62,830 \\
\hline \multicolumn{2}{|c|}{ Summer } & & & & & & 103,498 & & 94,496 & & 102,168 \\
\hline Low Load & 50 & 337.8 & & 920 & & 0.050 & 15,597 & 272.5 & 12,581 & 337.1 & 15,563 \\
\hline Middle Load & 75 & 464.3 & & 736 & & 0.099 & 33,673 & 439.5 & 31,877 & 468.2 & 33,956 \\
\hline Full Load & 100 & 604.7 & & 552 & & 0.162 & 54,228 & 558.0 & 50,039 & 587.1 & 52,648 \\
\hline \multicolumn{2}{|c|}{ Spring/Autumn } & & & & & & 82,641 & & 71,094 & & 86,554 \\
\hline Low Load & 25 & 269.1 & & 1530 & & 0.050 & 20,660 & 158.8 & 12,189 & 257.3 & 19,755 \\
\hline Middle Load & 50 & 285.3 & & 1224 & & 0.070 & 24,541 & 261.5 & 22,493 & 323.9 & 27,856 \\
\hline Full Load & 75 & 444.2 & & 918 & & 0.092 & 37,440 & 432.0 & 36,413 & 462.0 & 38,943 \\
\hline \multicolumn{2}{|c|}{ Winter } & & & & & & 57,516 & & 65,058 & & 74,628 \\
\hline Low Load & 25 & 91.4 & & 1200 & & 0.057 & 6,233 & 130.0 & 8,864 & 171.8 & 11,713 \\
\hline Middle Load & 50 & 169.4 & & 960 & & 0.099 & 16,059 & 191.0 & 18,103 & 249.4 & 23,637 \\
\hline Full Load & 75 & 346.1 & & 720 & & 0.141 & 35,225 & 374.3 & 38,092 & 385.9 & 39,279 \\
\hline \multicolumn{2}{|c|}{ Total } & & & 8760 & & & 308,370 & & 290,364 & & 326,180 \\
\hline
\end{tabular}




\section{Summary}

The modular concept centrifugal chiller has lots of opportunities to save energy by the helps of series counter flow effect and compressor sequence operation to the loads. The study says that the modular concept chiller with uneven capacities offers $24 \%$ improved IPLV and $6 \%$ COP at AHRI condition compared to a conventional single compressor without inverter. If it compared with dual compressor chiller, it has $8.7 \%$ better IPLV. A centrifugal chiller has a dynamic compressor, its benefits by inverter should be limited by the condition of operation heads at site. The energy performance of the modular concept centrifugal chiller is competitive when it compares with a conventional single compressor equipped an inverter, but the modular concept chiller always provides better COPs at all load conditions based on CCET as shown in table 5. The modular concept centrifugal chiller has shown comparable opportunities for energy savings at the most of the weather and load conditions.

\section{References}

1. Hartmut Krain, Review of Centrifugal Compressor's Application and Development, Journal of Turbomachinery, Vol. 127, pp. 25 34.(2005)

2. KEPCO, http://www.kepco.co.kr

3. Joost Brasz, Lee Tetu, Variable-Speed Centrifugal Chiller Control for variable primary flow(VRF) applications, International Refrigeration and Air Conditioning Conference at Purdue, (2008)

4. ASHRAE, ASHRAE Handbook 2000 HVAC Systems and Equipment(2000) 